\title{
АНАЛИТИЧЕСКИЙ КОНТРОЛЬ МАССОВОЙ ДОЛИ ОСНОВНОГО ВЕЩЕСТВА В АНТИОКСИДАНТАХ 2,4,6-ТРИ-трет-БУТИЛФЕНОЛ И ПЕНТАЭРИТРИТОВЫЙ ЭФИР 3,5-ДИ-трет-БУТИЛ-4-ГИДРОКСИФЕНИЛПРОПИОНОВОЙ КИСЛОТЫ МЕТОДОМ ВЫСОКОЭФФЕКТИВНОЙ ЖИДКОСТНОЙ ХРОМАТОГРАФИИ
}

\author{
Г. В. Каликина, О.В. Шестакова, О.А. Возисова, \\ М. А. Леухина, И. Л. Исупова
}

\begin{abstract}
ГАЛИНА ВИКТОРОВНА КАЛИКИНА - начальник аналитической отдела АО «Научно-исследовательский институт полимерных материалов». Область интересов: аналитический контроль наполненных полимерных материалов, их компонентов и производственных вод с применением современных физикохимическихметодов анализа.E-mail: ustyuzhanina-gal@mail.ru.
\end{abstract}

ОЛЬГА ВЛАДИМИРОВНА ШЕСТАКОВА - инженер аналитической лаборатории АО «Научноисследовательский институт полимерных материалов». Область интересов: аналитический контроль наполненных полимерных материалов и их компонентов с применением современных физико-химических методов анализа. E-mail: shestakova.o.v@mail.ru.

ОЛЬГА АЛЕКСАНДРОВНА ВОЗИСОВА - начальник аналитической лаборатории АО «Научноисследовательский институт полимерных материалов». Область интересов: аналитический контроль наполненных полимерных материалов и их компонентов с применением современных физико-химических методов анализа.E-mail: olga.vozisova@mail.ru.

МАРИЯ АЛЕКСЕЕВНА ЛЕУХИНА - инженер аналитической лаборатории АО «Научно-исследовательский институт полимерных материалов». Область интересов: аналитический контроль наполненных полимерных материалов и их компонентов с применением современных физико-химических методов анализа.E-mail: leuhina.ma@mail.ru.

ИРИНА ЛЕОНИДОВНА ИСУПОВА - кандидат физико-математических наук, старший научный сотрудник отдела информационных технологий $A O$ «Научно-исследовательский институт полимерньх материалов». Область интересов: математическое моделирование и численные методы исследования полимерных систем; математическое моделирование неравновесных процессов воспламенения, горения, взрыва; методы обработки и анализа экспериментальной информации. E-mail: enotyforever@yandex.ru.

614113, Россия, г. Пермь, ул. Чистопольская, 16. АО «Научно-исследовательский институт полимерньх материалов».

Разработана методика определения массовой доли основного вещества в антиоксидантах 2,4,6-три-трет-бутилфенол (антиоксидант П-23) и пентаэритритовый эфир 3,5-ди-трет-бутил-4гидроксифенилпропионовой кислоты (антиоксидант Агидол-110) методом высокоэффективной жидкостной хроматографии.

Методика основана на растворении антиоксиданта в ацетонитриле с последующим добавлением этилового спирта, разделении основного вещзества и примесей на хроматографической колонке, записи хроматограммы и расчете массовой доли методом нормализации.

Методика, позволяющая определить массовую долю основного вещества в пределах для антиоксидантов П-23 (от 98,0 до $100 \%$ и Агидол-110 (от 95,0 до 100 \%), рекомендована для аналитического контроля 
массовой доли основного вещества в антиоксидантах при входном контроле сырья и после истечения гарантийного срока хранения.

Ключевые слова: высокоэффективная жидкостная хроматография, антиоксиданты, Агидол-110, Сонгнокс 1010, Ирганокс 1010, Эвернокс 10, П-23, ИК-спектроскопия, статистические математические модели.

\title{
ANALYTICAL CONTROL OF MASS FRACTION OF BASIC SUBSTANCE IN ANTIOXIDANTS 2,4,6-TRI-tert-BUTYLPHENOL AND PENTAERYTHRITOL ETHER 3,5-DI-tert-BUTYL-4- HYDROXYPHENYLPROPIONIC ACID BY HIGH-PERFORMANCE LIQUID CHROMATOGRAPHY
}

\author{
G. Kalikina, O. Shestakova, O. Vozisova, M. Leukhina, I. Isupova \\ 16, Chistopolskaya st., Perm, 614113, Russia. JSC “Research Institute of Polymeric Materials".
}

The technique of determining the mass fraction of the basic substance in antioxidants 2,4,6-tri-tert-butylphenol (antioxidant P-23) and pentaerythritol ether 3,5-di-tert-butyl-4-hydroxyphenylpropionic acid (antioxidant Agidol-110) is developed by high-performance liquid chromatography.

The technique is based on dissolution of the antioxidant in acetonitrile followed by the addition of ethyl alcohol, separation of the basic substance and impurities by column chromatography, record the chromatogram and calculate the mass fraction by normalization method.

The technique allows to determine mass fraction of basic substance in the range of antioxidant:

- P-23 - from 98,0 to $100 \%$;

- Agidol-110 - from 95,0\% to $100 \%$.

The developed method is recommended for analytical monitoring of the mass fraction of the basic substance in antioxidants during incoming inspection of raw materials, and after the expiration of the warranty period of storage.

Keywords: high performance liquid chromatography, antioxidants, Agidol-110, Songnox 1010, Irganox 1010, Evernox 10, P-23, IR-spectroscopy, statistical mathematical models.

Устойчивость полимера к действию кислорода обеспечивают антиоксиданты. Изучение кинетики окисления полимеров показало, что взаимодействие полимеров с кислородом протекает как радикальный цепной процесс, включающий стадии инициирования с образованием радикалов, развитие реакционной цепи, разветвление и обрыв цепи.

Роль антиоксидантов (ингибиторов) сводится к обрыву реакционной цепи окисления за счет взаимодействия с перекисным радикалом.

Среди антиоксидантов, обрывающих цепи, наиболее эффективными являются замещенные фенолы. Подвижный атом водорода фенолов легко отрывается и присоединяется к радикалу, обеспечивая обрыв цепи. При этом образуется гидроперекись, которая распадается на два радикала. Эти ра- дикалы рекомбинируют с образованием устойчивых соединений [1].

Антиоксидант Агидол-110 применяется для стабилизации бутилкаучука, а антиоксидант П-23 используется в качестве ингибитора полимеризации в материалах типа БК.

Антиоксидант П-23 представляет собой белый кристаллический порошок с возможным голубоватым, желтоватым или зеленоватым оттенком; практически не растворим в воде, хорошо растворим в органических растворителях (спиртах, углеводородах, эфире, ацетоне) [2].

Антиоксидант Агидол-110 - мелкий порошок от белого до светло-желтого цвета, хорошо растворим в бензоле, ацетоне, толуоле; малорастворим в спиртах; не растворим в воде [3]. 
Аналоги антиоксиданта Агидол-110 по CAS № 6683-19-8

\begin{tabular}{lcc}
\hline Торговое название & Фирма, страна-производитель & Структурная формула \\
\hline Ирганокс 1010 & «Сiba», Швейцария \\
Эвернокс 10 & Everspring Specialites Co., Ltd, Тайвань \\
Сонгнокс 1010 & Sonwon Industrial Co., Ltd, Корея & $\left.\left(\mathrm{CH}_{3}\right)_{3} \mathrm{C}\right)_{3} \mathrm{C}$
\end{tabular}

У антиоксиданта Агидол-110 существует ряд аналогов: Ирганокс 1010, Эвернокс 10, Сонгнокс 1010, которые приведены в таблице 1 [4].

Методом инфракрасной спектроскопии (ИКС) исследовали антиоксидант Агидол-110 и его аналоги на Фурье-спектрометре Nicolet iS50 FT-IR с помощью приставки нарушенного полного внутреннего отражения (НПВО) с алмазным кристаллом в области частот $4000 \div 400 \mathrm{~cm}^{-1}$ без предварительной пробоподготовки. Спектры исследуемых образцов сравнивали между собой и со спектрами приложенных к прибору библиотек.

Анализ спектров всех исследуемых образцов показал, что спектры образцов Сонгнокс 1010 и Эвернокс 10, а также Ирганокса 1010 и Аги- дола-110 идентичны друг другу и библиотечному спектру tetrakis [methylene(3,5-di-tert-butyl-4hydroxyhydrocinnamate)] methane.

Но совпадение с библиотечным спектром в случае антиоксидантов Сонгнокс 1010 и Эвернокс 10 составляет $80 \%$, а для Ирганокса 1010 и Агидола-110 - 70 \%. Спектры антиоксидантов Сонгнокса 1010 и Эвернокса 10 отличаются от спектра Ирганокса 1010 и Агидола-110 наличием дополнительных полос поглощения при частотах 905, 951, 1030 , 1300, 1722 и $3593 \mathrm{~cm}^{-1}$, а также перераспределением интенсивности некоторых полос поглощения в области частот от 1000 до $1300 \mathrm{~cm}^{-1}$.

Сравнивая спектры исследуемых соединений, можно предположить, что они содержат компо-

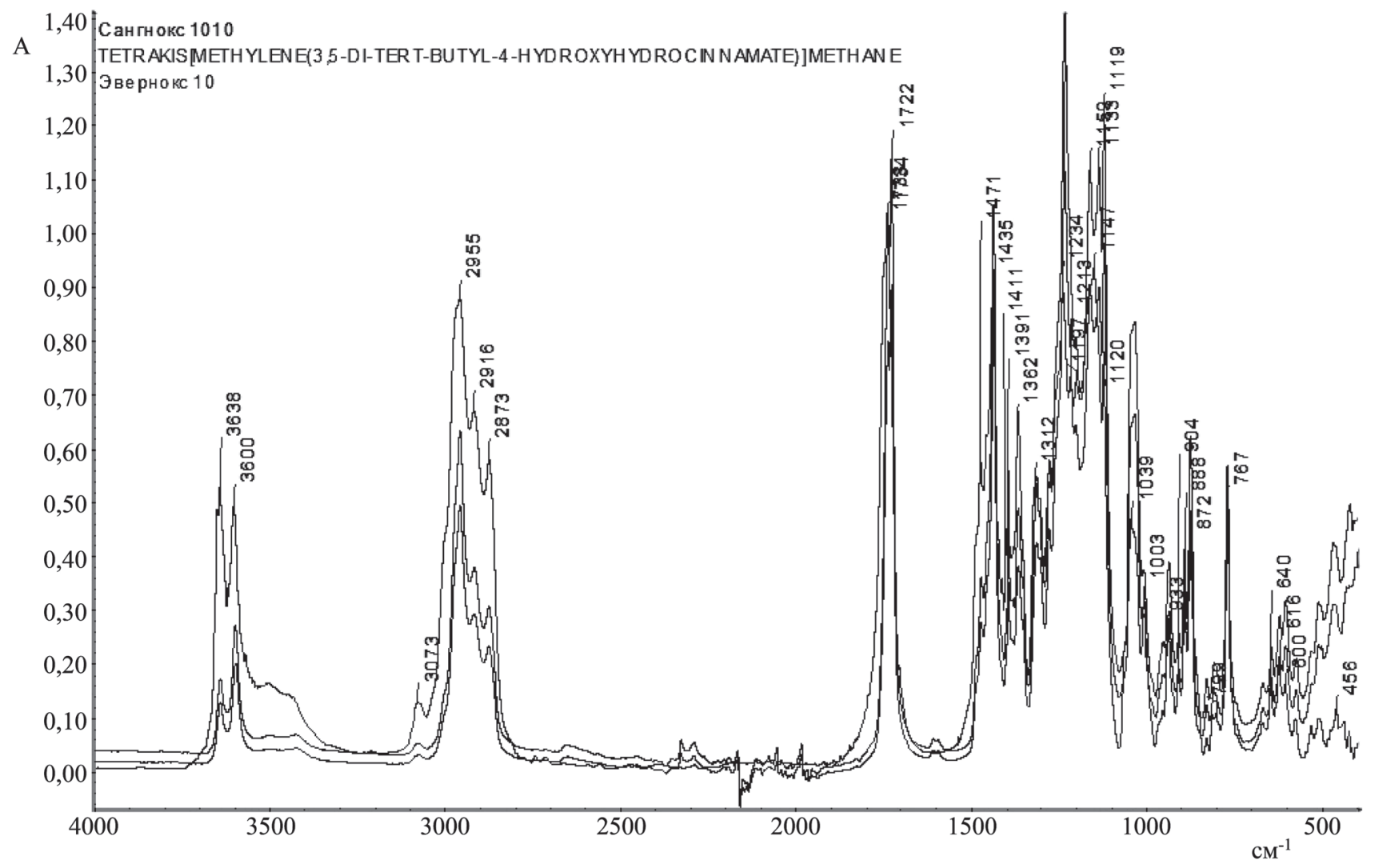

Рис. 1. Спектры образцов Эвернокса 10 и Сонгнокса 1010 и библиотечный спектр tetrakis [methylene(3,5-di-tert-butyl-4-hydroxyhydrocinnamate)] methane 


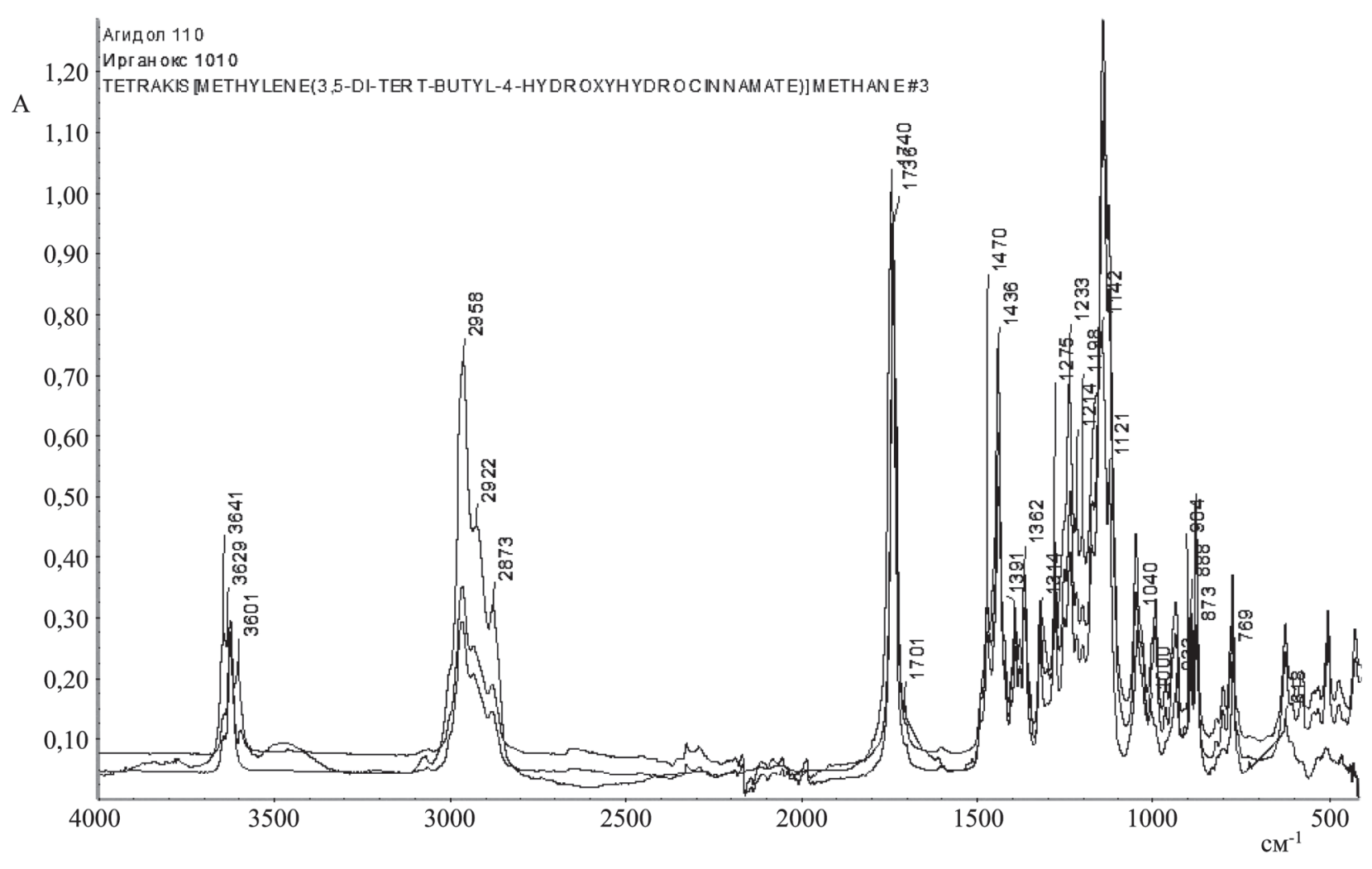

Рис. 2. Спектры образцов Ирганокса 1010 и Агидола-110 и библиотечный спектр tetrakis [methylene(3,5-di-tert-butyl-4-hydroxyhydrocinnamate)] methane

ненты того же класса соединений-антиоксидантов, но структура их несколько отличается, в связи с чем наблюдается наличие дополнительных полос поглощения, а также перераспределение интенсивности.

Техническими условиями ТУ 2425-03005807999-2002 Антиоксидант П-23 не нормируется содержание основного вещества, которое является необходимым критерием оценки качества продукта.

Для контроля качества антиоксиданта Агидол-110 используются технические условия ТУ 2492-447-05742686-2006, в соответствии с требованиями которых методом жидкостной хроматографии определяется массовая доля основного вещества. В связи с прекращением производства хроматографической колонки «типа Luna 5u c OOD 42520 - EO», «фаза Нурersil» массовую долю основного вещества в антиоксидантах Агидол-110, Ирганокс 1010, Эвернокс 10, Сонгнокс 1010 определяли на альтернативной колонке «ZORBAX Eclipse XDB-C18».

\section{Определение антиоксиданта Агидол-110 и П-23 методом высокоэффективной жидкостной хроматографии}

Условием применения метода является хорошая растворимость исследуемого компонента в растворителе (элюенте). В процессе хроматографирования компоненты не претерпевают химических превращений, разделение происходит вследствие различной скорости движения хроматографических зон компонентов в потоке жидкости вдоль колонки [5].

Определение антиоксиданта Агидол-110 и П-23 проводили на жидкостном хроматографе “Agilent 1200" с диодно-матричным детектором и автоматическим вводом пробы. Сбор информации и обработка результатов осуществлялись с помощью программного обеспечения "ChemStation". Для определения массовой доли антиоксиданта П-23 и Агидол-110 реализован вариант обращеннофазовой хроматографии (ОФХ).

Для подбора оптимальных условий хроматографического определения массовой доли основного вещества в антиоксидантах были построены статистические математические модели (регрессионные соотношения между входными и выходными параметрами) на основе экспериментального исследования [6,7].

В качестве входных параметров были выбраны следующие условия:

- концентрация элюента «Ацетонитрил» $\left(X_{1}\right)$;

- температура термостата колонки $\left(X_{2}\right)$. 
В качестве выходных факторов были рассмотрены следующие хроматографические параметры для компонентов Агидол-110, Ирганокс 1010, Эвернокс 10, Сонгнокс 1010 и П-23:

- время удержания $\left(Y_{1}-Y_{5}\right.$ соответственно);

- площадь пика ( $Y_{6}-Y_{10}$ соответственно);

- содержание вещества по методу нормализации ( $Y_{11}-Y_{15}$ соответственно);

- чувствительность вещества в элюенте $\left(Y_{16}-Y_{20}\right.$ соответственно).

Для установления формы зависимости между входными факторами и откликом, выделения выбросов был проведен предварительный графический анализ экспериментальных данных. В результате анализа было установлено, что выходные параметры в основном нелинейно зависят от первого аргумента, поэтому был выбран следующий вид уравнения регрессии:

$$
Y=b_{0}+b_{1} X_{1}+b_{2} e^{-b_{3} X_{1}}+b_{4} X_{2},
$$

где $b_{0}-$ свободный член уравнения регрессии; $b_{l}$, $b_{2}, b_{3}, b_{4}-$ коэффициенты уравнения регрессии.

Расчет был проведен по программе множественного регрессионного анализа, реализующей метод Эфроймсона.

Зависимости времени удержания Агидола-110, Ирганокса 1010, Эвернокса 10, Сонгнокса 1010 от соотношения элюента «ацетонитрил : вода» и температуры термостата колонки не выявлено. В этом случае возможно влияние других факторов, не включенных в регрессионную модель. Зависимость времени удержания антиоксиданта П-23 от рассматриваемых факторов имеет следующий вид:

$Y_{5}=107,456957-0,868957 X_{1}+158,808066 e^{-0,022 X_{1}}$,

$R^{2}=0,88906$,

где $R^{2}$ - множественный коэффициент детерминации, который используется для оценки общего качества уравнения регрессии и показывает степень соответствия между исходными данными и регрессионной моделью (может принимать значения от 0 до 1). Таким образом, величина $1-R^{2}$ определяет, сколько вариаций обусловлено факторами, не включенными в модель. По общим рекомендациям, если $R^{2} \geq 0,75$, то модель можно использовать для дальнейшего анализа данных.

Стоит отметить, что для всех построенных регрессионных уравнений зависимости хроматографических параметров от температуры термостата колонки не выявлено.

Для определения оптимальных условий хроматографирования можно использовать зависимо- сти чувствительности вещества в элюенте, представленные в виде линий равного уровня для поверхностей $\mathrm{Y}_{16-20}\left(\mathrm{X}_{1}, \mathrm{X}_{2}\right)$. По приведенным графикам для заданной степени чувствительности можно определить оптимальное значение концентрации элюента.

По приведенным графикам (рис. 3,4 ) и в ходе экспериментов были подобраны оптимальные ус-

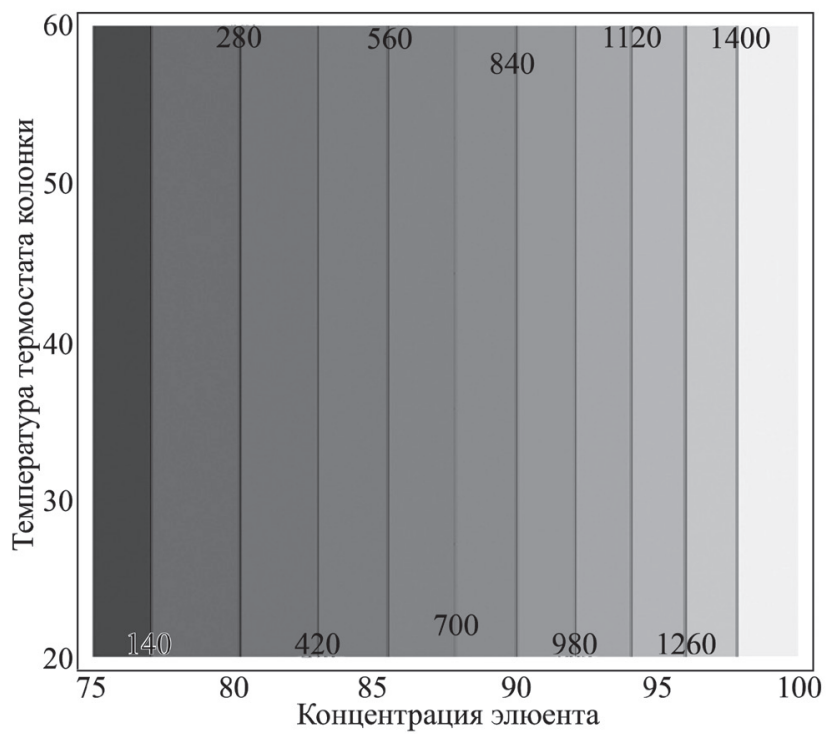

Рис. 3. Линии уровня для поверхности, описывающей зависимость чувствительности вещества в элюенте для Агидола-110 от концентрации элюента и температуры термостата колонки

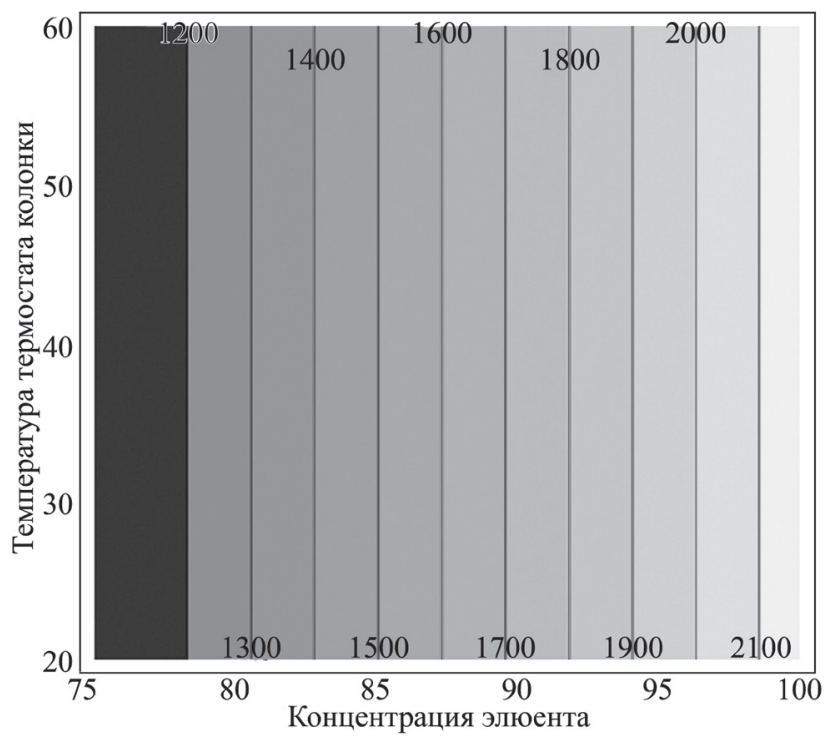

Рис. 4. Линии уровня для поверхности, описывающей зависимость чувствительности вещества в элюенте для П-23 от концентрации элюента и температуры термостата колонки 
ловия определения массовой доли основного вещества в антиоксидантах:

- в качестве неподвижной фазы применен модифицированный силикагель ZORBAX Eclipse $\mathrm{XDB}-\mathrm{C} 18$;

- в качестве подвижной фазы (элюента) использован ацетонитрил $100 \%$;

- расход элюента $1 \mathrm{~cm}^{3} /$ мин;

- детектирование проведено при длине волны 220 нм;

- температура термостата $40{ }^{\circ} \mathrm{C}$;

- объем вводимой пробы 5 мкдм³

- время анализа составляет 15 мин.

На всех хроматограммах можно наблюдать хорошо разделенные и симметричные пики основного вещества и примесей.

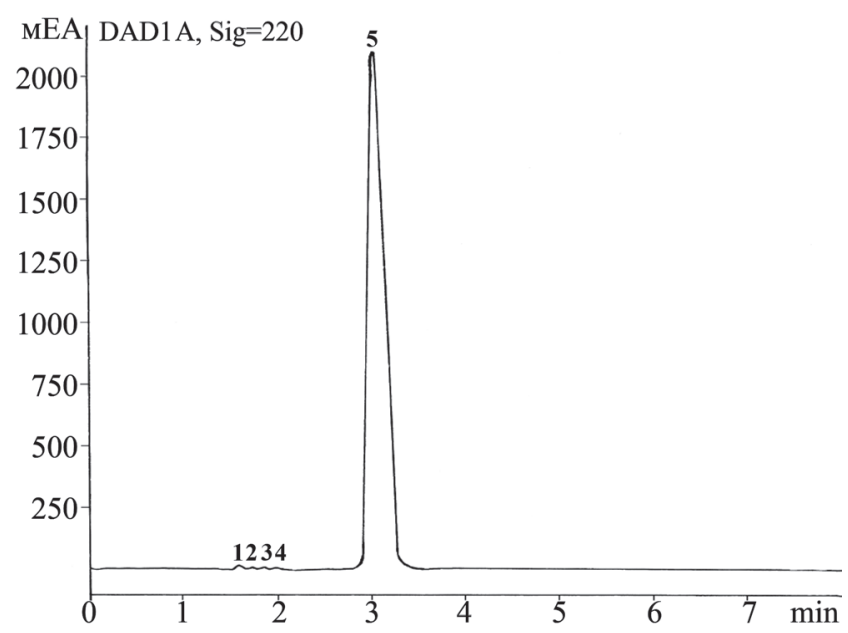

Рис. 5. Хроматограмма антиоксиданта П-23:

1, 2,3,4-неидентифицированнье примеси, 5 - основное вещество

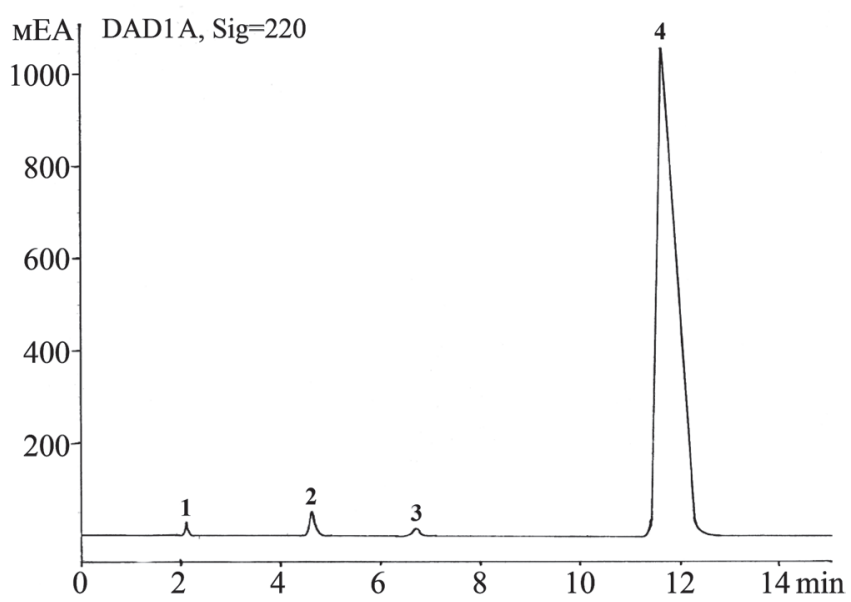

Рис. 6. Хроматограмма антиоксиданта Агидол-110:

1, 2,3-неидентифицированные примеси, 4 - основное вещество
Вид хроматограмм антиоксиданта П-23 и Агидол-110 представлен на рисунках 5 и 6.

Для оценки метрологических характеристик методики были проанализированы производственные образцы, оценка показателей качества проведена в соответствии с рекомендациями по межгосударственной стандартизации [8].

В табл. 2 и 3 приведены результаты анализа образцов антиоксидантов П-23 и Агидол-110.

Таблица 2

Результаты определения массовой доли основного вещества в антиоксиданте П-23

\begin{tabular}{cccc}
\hline \multirow{2}{*}{$\begin{array}{c}\text { обомер } \\
\text { образца }\end{array}$} & \multicolumn{2}{c}{ Результат определения, $\%$} & $\mathbf{X i}$ \\
\cline { 2 - 3 } & $\mathbf{X}_{\mathbf{1}}$ & $\mathbf{X}_{\mathbf{2}}$ & \\
\hline 1 & 99,45 & 99,58 & 99,52 \\
3 & 99,51 & 99,52 & 99,51 \\
4 & 99,42 & 99,40 & 99,41 \\
5 & 99,39 & 99,39 & 99,39 \\
6 & 99,54 & 99,54 & 99,54 \\
7 & 99,42 & 99,48 & 99,45 \\
8 & 99,38 & 99,40 & 99,39 \\
9 & 98,98 & 99,03 & 99,01 \\
10 & 98,15 & 98,08 & 98,12 \\
\hline
\end{tabular}

Таблиия 3

Результаты определения массовой доли основного вещества в антиоксиданте Агидол-110

\begin{tabular}{cccc}
\hline \multirow{2}{*}{$\begin{array}{c}\text { Номер } \\
\text { образца }\end{array}$} & \multicolumn{2}{c}{ Результат определения, $\%$} & $\mathbf{X}$ \\
\cline { 2 - 3 } & $\mathbf{X}_{\mathbf{1}}$ & $\mathbf{X}_{\mathbf{2}}$ & \\
\hline 1 & 97,60 & 97,51 & 97,56 \\
2 & 97,61 & 97,51 & 97,56 \\
3 & 96,57 & 96,58 & 96,58 \\
4 & 96,57 & 96,56 & 96,57 \\
5 & 96,95 & 96,97 & 96,96 \\
6 & 96,92 & 97,26 & 97,09 \\
7 & 98,43 & 98,41 & 98,42 \\
8 & 98,50 & 98,32 & 98,41 \\
9 & 97,53 & 97,51 & 97,52 \\
10 & 97,32 & 97,64 & 97,48 \\
\hline
\end{tabular}

Установлено, что систематическая погрешность определения отсутствует. Абсолютная погрешность определения для антиоксиданта П-23 составляет $\pm 1,00 \%$, для Агидол- $110 \pm 1,50 \%$. 


\section{Выводы}

1. Изучены антиоксиданты методом инфракрасной спектроскопии на Фурье-спектрометре Nicolet iS50 FT-IR с помощью приставки НПВО с алмазным кристаллом в области частот $4000 \div 400 \mathrm{~cm}^{-1}$. Показано, что спектры образцов Сонгнокс 1010 и Эвернокс 10, а также Ирганокс 1010 и Агидол- 110 идентичны друг к другу и библиотечному спектру tetrakis[methylene(3,5-ditert-butyl-4-hydroxyhydrocinnamate)] methane.

2. Изучено влияние параметров хроматографической системы на определение антиоксидантов. Установлено, что для заданной степени чувствительности можно установить оптимальное значение концентрации элюента.

3. Для определения массовой доли основного вещества в антиоксидантах П-23 и Агидол-110 использован метод высокоэффективной жидкостной хроматографии, с помощью которого разработана методика определения массовой доли основного вещества в антиоксидантах П-23 и Агидол-110, рекомендованная для аналитического контроля массовой доли основного вещества в антиоксидантах при входном контроле сырья и после истечения гарантийного срока хранения.

Диапазон измеряемой массовой доли антиоксиданта П-23 находится в пределах от 98,0 до $100 \%$. Абсолютная погрешность определения при вероятности $\mathrm{P}=0,95$ составляет \pm 1,0 \%. Допустимое расхождение между парал- лельными определениями составляет $0,15 \%$. Абсолютная погрешность определения для антиоксиданта Агидол-110 при вероятности $\mathrm{P}=$ 0,95 составляет $1,5 \%$. Допустимое расхождение между параллельными определениями составляет $0,32 \%$.

\section{Литература}

1. Рогов Н.Г. Смесевые ракетные твердые топлива: Компоненты. Требования. Свойства / Н.Г. Рогов, М.А. Ищенко - Санкт-Петербург: СПбГТИ (ТУ), 2005.

2. Антиоксидант П-23: технические условия ТУ 2425030-05807999-2002/ ФГУП «НЗСП» / гл.инж. Олешко П.Р. - Новочеркасск, 2002.

3. Агидол-110: технические условия ТУ 2492-44705742686-2006/ ОАО «СНХЗ», рук. Кутузов П.И. Стерлитамак, 2006.

4. COMMON CHEMISTRY.A CASSOLUTION [электронный ресурc]:URL:http://www.commonchemistry. org. Дата обращения 02.10.2019.

5. Сычев К.С. Практическое руководство по жидкостной хроматографии. М: Техносфера, 2010.

6. Дрейпер Н., Смит Г. Прикладной регрессионный анализ. Книга 1. В 2-х кн. М.: Финансы и статистика, 1986.

7. Дрейпер Н., Смит Г. Прикладной регрессионный анализ. Книга 2. В 2-х кн. М.: Финансы и статистика, 1986.

8. Рекомендации по международной стандартизации РМГ61-2010 Показатели точности, правильности, прецизионности методик количественного химического анализа. Методы оценки. - М: Стандартинформ, 2012. 